\title{
Corrosion Evaluation of Modified Reinforcement Concrete by Electrochemical Noise Techniques
}

\author{
R.E. Núñez-Jaquez, ${ }^{\text {,,* }}$ C. Gaona-Tiburcio, ${ }^{a}$ J. Uruchurtu-Chavarin, \\ C.P. Barrios-Durstewitz, ${ }^{\text {a }}$ F. Almeraya-Calderón, ${ }^{a}$ A. Martínez-Villafañe ${ }^{a}$ \\ ${ }^{a}$ Centro de Investigación en Materiales Avanzados, S.C. Miguel de Cervantes No. 120, \\ Complejo Industrial Chihuahua, C.P. 31109, Chihuahua, Chihuahua, México \\ ${ }^{b}$ Centro de Investigación en Ingeniería y Ciencias Aplicadas, UAEM, Av. Universidad 1001, \\ C.P. 62210 Cuernavaca, Morelos, México
}

Received 19 April 2004; accepted in revised form 22 September 2004

\begin{abstract}
The reinforced concrete is a versatile and resistant material and it is used in several construction projects; for this reason the improvement of concrete properties and protection of the reinforced steel against corrosion is an active research area. Diverse methods are applied on the steel or on the concrete to protect them. The present investigation is directed towards the development and performance evaluation against corrosion of the reinforcement concrete, containing two different fly ash and blast furnace slag mixtures, in saline environments, and the corrosive evaluation was performed by electrochemical noise technique. The results show an improvement of the steel reinforcement corrosion resistance.
\end{abstract}

Keywords: fly ash, slag blast furnace, ternary blend, electrochemical noise.

\section{Introducción}

En la mayoría de los concretos comercializados en estos días, existe una gran variedad de puzolanas y escorias disponibles para su uso en concretos con resistencias regulares y altas. Recientemente, concretos que contienen más de

\footnotetext{
* Corresponding author. E-mail address: rosalba.nunez@cimav.edu.mx
} 
uno de estos materiales han llegado a ser muy comunes, hasta el punto de llegar a estar disponibles mezclas ternarias o cuaternarias.

El incorporar materiales cementantes suplementarios al cemento portland presenta grandes ventajas, debido a que desarrolla excelentes propiedades mecánicas y características de larga durabilidad. Además, el uso de estos materiales es beneficioso para el ambiente, debido a que el cemento es reemplazado parcialmente por estos [1].

Los materiales cementantes son sustancias que por sí solas tienen propiedades hidráulicas cementantes (fraguan y endurecen en presencia del agua). Los materiales cementantes incluyen a la escoria de alto horno, al cemento natural, a la cal hidráulica hidratada, y a las combinaciones de estos y de otros materiales. Las puzolanas son materiales silíceos que poseen una elevada capacidad acusada de cementación. Cuando dichos materiales están finamente molidos y húmedos, reaccionan con los hidróxidos de calcio a temperaturas ordinarias para formar compuestos con propiedades cementantes, que son silicatos monocálcicos de muy baja solubilidad; ejemplos de estos son las cenizas volantes, la microsilica y el metacaolín [7].

Investigaciones han demostrado que la proporción óptima de puzolana en una mezcla de cemento portland y puzolana, esta en el rango de $10 \%$ a $35 \%$ en peso de cemento más puzolana, dependiendo de la calidad de los materiales empleados.

Al mismo tiempo reemplazar cemento con escoria al 50\% por masa es apropiado por economía, sin embargo no es apropiado en mezclas binarias donde la durabilidad es importante.

A partir de varios estudios, se han comprobado las buenas propiedades y grandes ventajas de adicionar el cemento con algún tipo de puzolana o escoria $[2,3,4,5$, $6]$.

Generalmente las pruebas que se han elaborado en concretos de este tipo de mezclas son desde el punto de vista estructural (resistencia a la compresión) y funcional (permeabilidad, resistencia a los sulfatos, etc.). Es por esta razón que surge la necesidad de complementar estos estudios desde el punto de vista 
corrosivo; debido a lo anterior, el objetivo de este trabajo es realizar una evaluación de concretos que contengan materiales puzolánicos y escoria por medio de las técnicas electroquímicas.

Para el proceso experimental se prepararon dos tipos de especímenes cilíndricos adicionados con cenizas volantes y escoria de alto horno, con dos varillas de acero embebidas, los cuales se colocaron en un medio salino (agua con sal al 5\%) durante un período de tiempo; se observó cual fue el comportamiento corrosivo por medio del ruido electroquímico. En los resultados obtenidos se muestra cual es el comportamiento de cada mezcla.

Cuando transcurrió el tiempo de curado (28 días) se realizó la prueba de resistencia a la compresión a los especimenes, obteniendo valores superiores a los esperados.

\section{Desarrollo experimental}

\section{Especímenes de concreto}

Se elaboraron especímenes de concreto con cemento ordinario portland I, a los cuales se les sustituyó los siguientes porcentajes por peso de cemento con los aditivos: $5 \%$ de cenizas volantes y 10\% de escoria de alto horno; $10 \%$ de cenizas volantes y $5 \%$ de escoria de alto horno.

Las dimensiones de dichos especímenes son de $0.30 \mathrm{~m}$ de altura y $0.15 \mathrm{~m}$ de diámetro, con una relación $\mathrm{a} / \mathrm{c}$ de $0.45 \mathrm{y}$ una resistencia esperada de $250 \mathrm{~kg} / \mathrm{cm}^{2}$. A cada espécimen se les colocaron 2 varillas $0.00095 \mathrm{~m}$ de diámetro y $0.32 \mathrm{~m}$ de longitud, de acero 1018, las cuales sobresalen $0.02 \mathrm{~m}$ de la superficie del concreto, para ser empleadas en la prueba electroquímica y el mapeo de potenciales.

\section{Ruido electroquímico}

Para la aplicación de la técnica de ruido electroquímico, se tomaron la lectura de 4096 puntos con una velocidad de un punto por segundo. 


\section{Mapeo de potenciales}

Para realizar el mapeo de potenciales se efectuó de acuerdo a la norma ASTM C 876, a cada espécimen se le trazo un mallado con la finalidad de determinar en cada punto el potencial (Fig. 1).

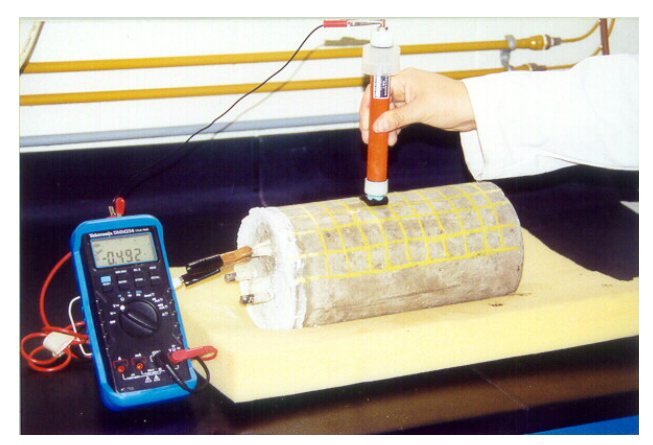

Figura 1. Mapeo de potenciales.

\section{Resultados y analisis de resultados}

A continuación se muestran los resultados obtenidos del mapeo de potenciales. El análisis de estas gráficas se realiza de acuerdo a los criterios de evaluación indicados en la norma ASTM C 876 (Tabla 1).

Tabla 1. Análisis de resultados en el mapeo de potenciales de acuerdo a la norma ASTM C876.

\begin{tabular}{c|c}
\hline \hline Potencial Ecorr $(\boldsymbol{V})$ & Riesgo de daño \\
\hline \hline Más positivos que -0.200 & $10 \%$ de probabilidad de corrosión \\
\hline-0.200 a -0.350 & Cierta incertidumbre \\
\hline Más negativos que -0.350 & $90 \%$ de probabilidad de corrosión \\
\hline \hline
\end{tabular}

De acuerdo a los resultados obtenidos en las gráficas, se puede observar que los especímenes con 5\% de cenizas y $10 \%$ de escoria (Fig. 2a-2d), presentan valores menores de potencial con respecto a los especímenes que contiene $10 \%$ de cenizas y 5\% de escoria (Fig. 3a-3d), por lo que la probabilidad de presencia de corrosión es pequeña. En general los dos tipos de mezclas presentan el mismo 
comportamiento, inician con valores que se encuentran en un rango de cierta incertidumbre (Fig. 2a y 3a) y posteriormente se presenta un alto riesgo de corrosión (Fig. 2c y 3c), para terminar de nuevo en el rango de cierta incertidumbre. La que menos problemas de corrosión presentó fue la mezcla que contiene $5 \%$ de cenizas y $10 \%$ de escoria a los 2 meses de exposición en el medio salino (Fig. 2b), ya que su rango se localizo entre -0.100 a $-0.250 \mathrm{~V}(10 \%$ probabilidad de corrosión).

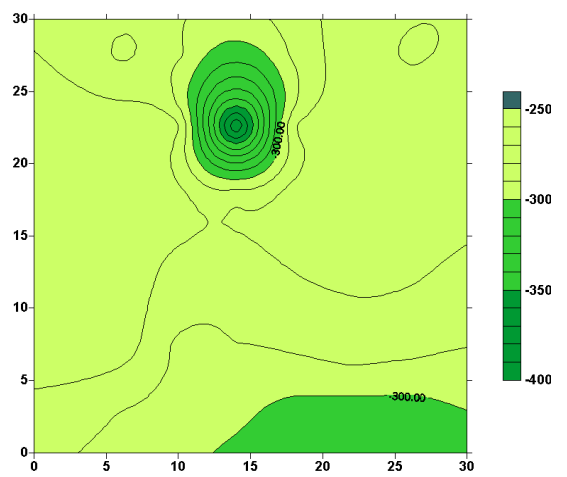

a) 0 días en medio salino.

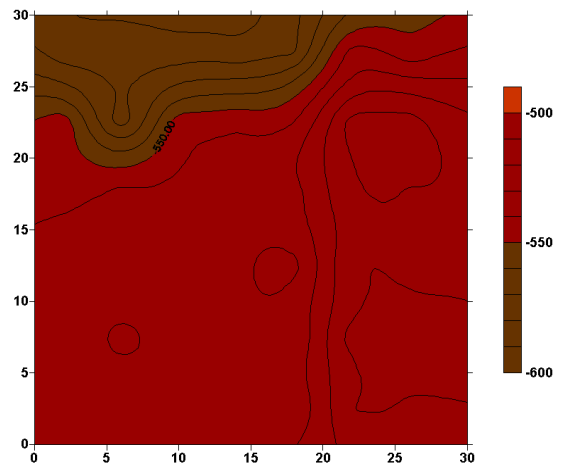

c) 5 meses en medio salino.

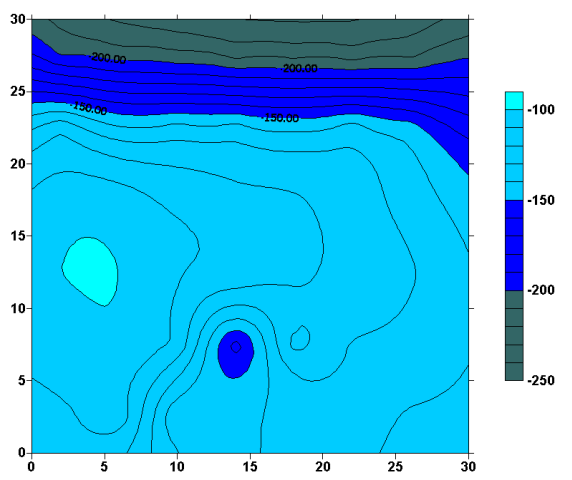

b) 2 meses en medio salino.

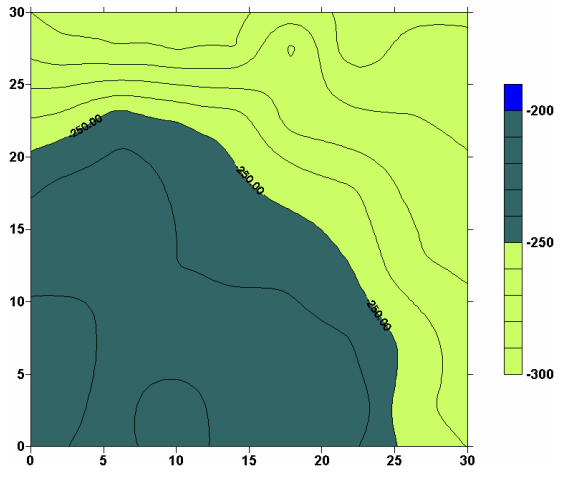

d) 10 meses en medio salino.

Figura 2. Mapeo de potenciales para las mezclas con 5\% de cenizas y $10 \%$ de escoria (a-d). 


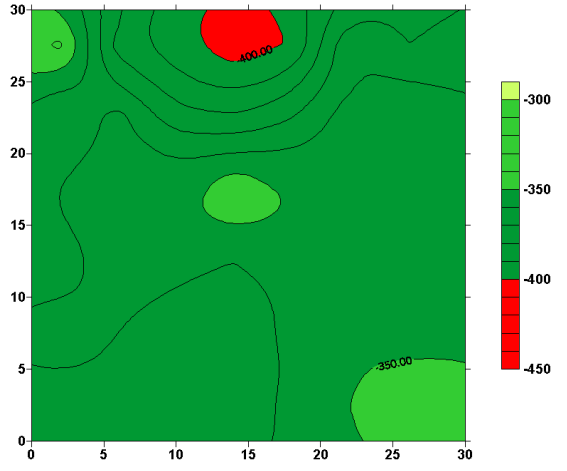

a) 0 días en medio salino.

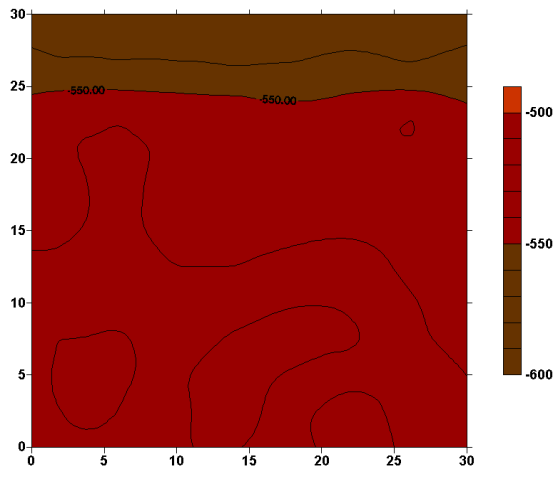

c) 5 meses en medio salino.

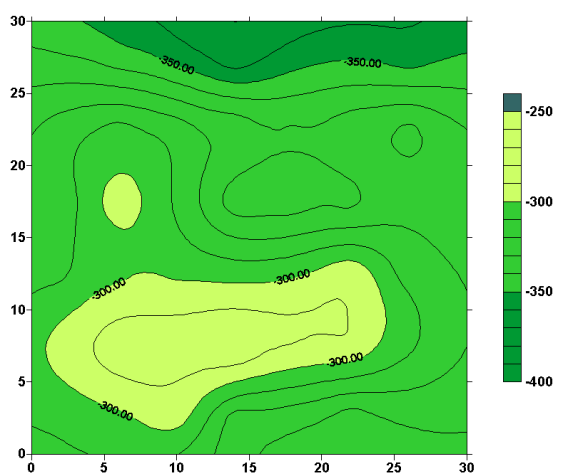

b) 2 meses en medio salino.

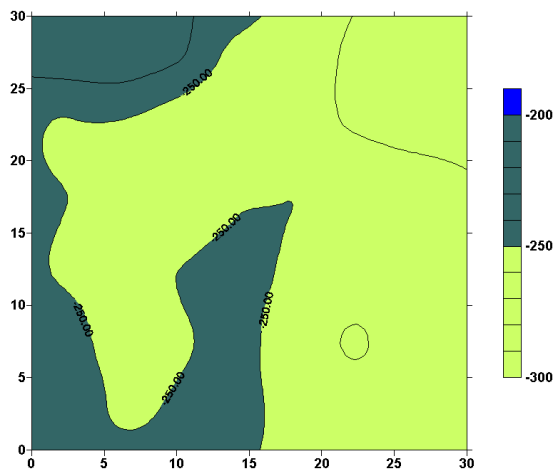

d) 10 meses en medio salino.

Figura 3. Mapeo de potenciales para las mezclas con $10 \%$ de cenizas y $5 \%$ de escoria (a-d).

En la tabla 2 se resumen los resultados obtenidos del análisis estadístico de las series de tiempo de potencial y corriente en la técnica de ruido electroquímico.

La resistencia de ruido $R_{n}$ se define como:

$$
R_{n}=\sigma_{V} / \sigma_{I}
$$

donde $\sigma_{V}$ es la desviación estándar del ruido del potencial y $\sigma_{I}$ es la desviación estándar del ruido de la corriente.

Posteriormente los resultados de la $i_{c o r r}$ se obtuvieron de la ecuación:

$$
i_{\text {corr }}=\frac{\beta}{R_{n}}
$$


Tabla 2. Resultados obtenidos del análisis estadístico.

\begin{tabular}{|c|c|c|c|}
\hline $\begin{array}{c}\text { Porcentaje de material } \\
\text { sustituido* }\end{array}$ & $\begin{array}{c}\text { Tiempo de exposición } \\
\text { en el medio salino }\end{array}$ & $\mathbf{R}_{\mathbf{n}}\left(\mathbf{\Omega}-\mathbf{c m}^{\mathbf{2}}\right)$ & $\mathbf{I}_{\mathbf{c o r r}}\left(\mathbf{A} / \mathbf{m}^{\mathbf{2}}\right)$ \\
\hline \multirow{3}{*}{$5 \%$ cenizas volantes } & 0 & 292130.0 & 0.0008 \\
\cline { 2 - 4 } & 2 meses & 2890.9 & 0.0899 \\
\cline { 2 - 4 } 10\% escoria de alto horno & 5 meses & 399.9 & 0.6501 \\
\cline { 2 - 4 } & 8 meses & 9878.5 & 0.0263 \\
\hline & 10 meses & 12615.0 & 0.0206 \\
\hline \multirow{3}{*}{$10 \%$ cenizas volantes } & & & 0.0450 \\
\cline { 2 - 4 } & 0 & 5767.1 & 0.3034 \\
\cline { 2 - 4 } $5 \%$ escoria de alto horno & 2 meses & 856.7 & 0.2459 \\
\cline { 2 - 4 } & 5 meses & 1057.0 & 0.1373 \\
\cline { 2 - 4 } & 8 meses & 1893.6 & 0.0177 \\
\hline
\end{tabular}

*Porciento sustituido con respecto al peso del cemento.

Con referencia a los valores de $\mathrm{i}_{\text {corr }}$ proporcionados por DURAR [9] (ver tabla 3) se puede realizar el análisis de los resultados anteriores.

Tabla 3. Clasificación de $i_{\text {corr }}$ en términos de vida útil.

\begin{tabular}{c|c}
\hline \hline$I_{\text {corr }}\left(\mathrm{A} / \mathrm{m}^{2}\right)$ & Nivel de corrosión \\
\hline \hline$<0.001$ & Despreciable \\
\hline $0.001-0.005$ & Moderado \\
\hline $0.005-0.1$ & Elevada \\
\hline$>0.1$ & Muy elevada \\
\hline \hline
\end{tabular}

De acuerdo a los resultados anteriores encontramos que la mezcla con mayores $i_{\text {corr }}$ es la que contiene $10 \%$ de ceniza volante y $5 \%$ de escoria de alto horno, pero a largo plazo presenta menores velocidades de corrosión que la de 5\% de cenizas volantes y $10 \%$ de escoria de alto horno.

A continuación se muestran algunas de las gráficas obtenidas de las series de tiempo de potencial y corriente en los especímenes (Fig. 4): 


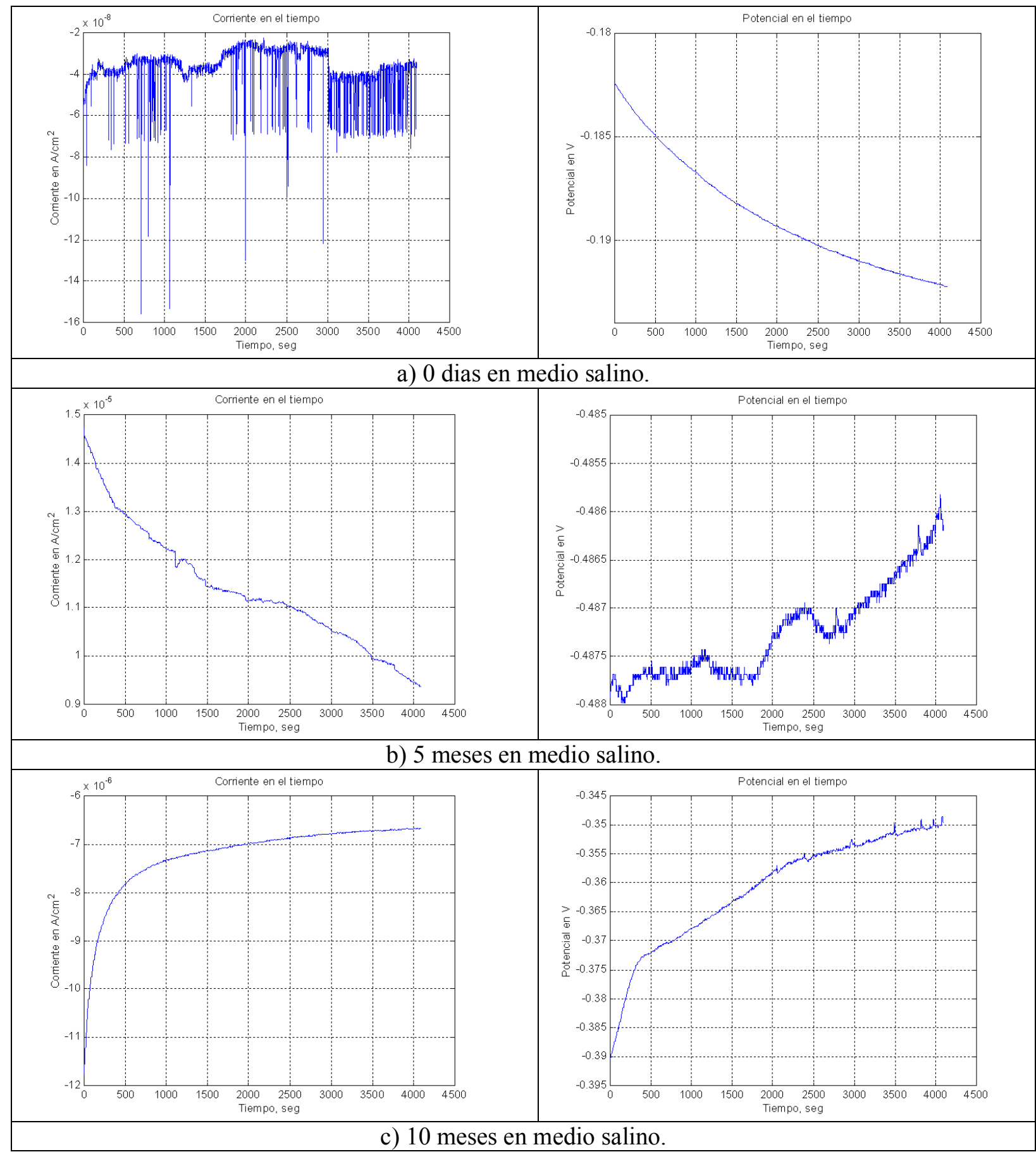

Figura 4. Series de tiempo de potencial y corriente para las mezcla con $5 \%$ de cenizas y $10 \%$ de escoria.

En la Fig. 4 se observan las gráficas de las series de tiempo de potencial y corriente obtenidas; en ellas se aprecia como son congruentes los resultados de las velocidades de corrosión obtenidas con los mapeos de potenciales. 
En la Fig. 4a se muestran las series de tiempo para potencial y corriente: en la de potencial se observan valores pequeños, además que estos se hacen más positivos, lo que nos indica poca probabilidad de corrosión, además que en la de corriente no se observa ninguna señal de picaduras, probablemente la formación de la capa pasiva.

En la Fig. 4b se aprecia en la serie de potencial un aumento de los valores de potencial, y en la corriente se aprecia un pequeño pico, lo cual talvez sea indicativo de la presencia de formación de alguna picadura.

Por último en la Fig. 4c se aprecia que la corrosión se mantiene elevada ya que el potencial y la demanda de corriente continúan aumentando.

\section{Conclusiones}

Desde el punto vista de las pruebas mecánicas, se aprecia que la diferencia del comportamiento entre una y otra mezcla es muy pequeña, por lo que se puede concluir que la mezcla que contiene $5 \%$ de cenizas volantes y $10 \%$ de escoria de alto horno es tan buena como la que contiene $10 \%$ de cenizas volantes y $5 \%$ de escoria de alto horno.

A partir de los resultados de la $i_{\text {corr }}$ se puede concluir que la mezcla que contiene $5 \%$ de cenizas volantes y 10\% de escoria de alto horno presenta mejores propiedades en contra de la corrosión, ya que es la que muestra valores más pequeños.

De las dos mezclas la que se pude señalar como mejor es la de 5\% de cenizas volantes y $10 \%$ de escoria de alto horno.

En los resultados se demuestra que no necesariamente una mezcla que tiene una buena resistencia puede presentar una buena protección en contra de la corrosión por agentes agresivos como la sal. 


\section{Evaluacion de la Corrosión en Concretos Adicionados con una Puzolana y Escoria por Medio del Ruido Electroquimico}

\section{Resumen}

Como ya es conocido, el concreto reforzado es un material versátil y resistente para su utilización en diversos campos, por ello se continúa con el mejoramiento de las propiedades del concreto y la protección del acero de refuerzo en contra de la corrosión; debido a lo anterior se han desarrollado diversos métodos que se aplican en el acero o en el concreto. En este último se han realizado modificaciones en su elaboración, adicionando materiales que le confieren resistencia y protección en contra del deterioro; algunos de estos materiales son las puzolanas (como las cenizas volantes) y la escoria, ya sea uno solo o en combinaciones de dos o tres materiales, observándose muy buenos resultados.

En este trabajo se hace una comparación por medio del ruido electroquímico, de concretos reforzados a los cuales se les añadieron cenizas volantes y escoria de alto horno, complementado la evaluación con los resultados obtenidos del mapeo de potenciales.

Para el proceso experimental se prepararon especímenes cilíndricos con dos proporcionamientos de cenizas volantes y escoria, y dos varillas de acero embebidas, los especímenes se colocaron en un medio salino (agua con sal al 5\%), y se observó el comportamiento corrosivo del acero por medio del ruido electroquímico.

Palabras claves: cenizas volantes, escoria de alto horno, puzolanas, mezclas ternarias, ruido electroquímico.

\section{Referencias}

1. P. Gu, J.J. Beaudoin, M. Zhang, V.M. Malhotra, Performance of Reforcing Steel in Concrete Containing Silica Fume and Blast Furnace Slag Pounded with Sodium Chloride Solution, ACI Materials Journal, 2000.

2. C. Ozyildrim, Resistance to Penetration of Chlorides into Concretes Containing Latex, Fly Ash, Slag, And Silica Fume, SP 145-26 ACI, Durability of Concrete $3^{\text {th }}$ International Conference, Nice, France, 2004.

3. H.T. Cao, M. Jedy, M. Rahimi, Properties of High Strength Concrete Using Cement Blended with Silica Fume, Fly Ash and Blast Furnace Slag, Concrete Institute of Australia, Concrete '89, Australia, Mayo de 1989. 
4. I. Hinczak, H. Roper, W. South, Ternary and Quaternary Cement Systems, Fly Ash, Silica Fume, Slag and Natural Pozzolans in Concrete, Supplementary Papers, 1992.

5. I. Hinczack, H. Roper, Ternary Cements, Short and Long-Term Properties, Concrete for the Nineties, Conference Proceedings, Australia, September 1990.

6. N.R. Short, C.L. Page, The Diffusion of Chloride Ions Trough Portland and Blended Cement Pastes, Silicates Industries, 1982.

7. Instructivo para concreto. Sría de Recursos Hidráulicos, México, 1960.

8. W.B. Butler, Durable Concrete Containing Three or Four Cementitious Materials, SP 170-15, Durability of Concrete, ACI, 1997.

9. O. Tróconis Gladis et al., Manual de Inspección, Evaluación y Diagnóstico de Corrosión en Estructuras de Hormigón Armado, DURAR, 1997. 